\title{
Molecular Heterogeneity of Head and Neck Squamous Cell Carcinoma Defined by Next-Generation Sequencing
}

\author{
Pan Zhang, Neena Mirani, Ada Baisre, and Helen Fernandes
}

From the Department of Pathology and Laboratory Medicine, Rutgers Biomedical and Health Sciences-New Jersey Medical School, Newark, New Jersey

\begin{abstract}
CME Accreditation Statement: This activity ("ASIP 2014 AJP CME Program in Pathogenesis") has been planned and implemented in accordance with the Essential Areas and policies of the Accreditation Council for Continuing Medical Education (ACCME) through the joint sponsorship of the American Society for Clinical Pathology (ASCP) and the American Society for Investigative Pathology (ASIP). ASCP is accredited by the ACCME to provide continuing medical education for physicians.
\end{abstract}

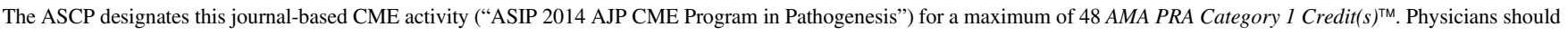
only claim credit commensurate with the extent of their participation in the activity.

CME Disclosures: The authors of this article and the planning committee members and staff have no relevant financial relationships with commercial interests to disclose.

\author{
Accepted for publication \\ January 2, 2014. \\ Address correspondence to \\ Helen Fernandes, Ph.D., \\ Department of Pathology and \\ Laboratory Medicine, Weill \\ Cornell Medical College, 1300 \\ York Ave., Room F-705, New \\ York, NY 10065. E-mail: \\ hef9020@med.cornell.edu.
}

\begin{abstract}
Head and neck squamous cell carcinoma (HNSCC) can be divided into two different clinical entities based on their association with high-risk subtypes of human papilloma virus (HPV16 and HPV18). Dissimilarities in prognosis and molecular profiles have attracted much attention in recent years, in part because of increasing rates of HPV infection in HNSCC; however, the underlying mechanisms and detailed genetic profiles that set these tumors apart are still elusive. To elucidate oncogenic pathways in HNSCC with and without HPV infection, we used targeted next-generation sequencing to interrogate single-nucleotide polymorphisms (SNPs) in 50 cancer-related genes. We detected SNPs in 25 of these genes from HNSCC tissue specimens with and without HPV infection. In 5 of the 25 genes, variant patterns were similar regardless of HPV infection status. A greater number of sequence variants in genes from the tyrosine kinase receptors and their associated pathways were preferentially present in $\mathrm{HPV}^{+}$ specimens. SNPs in genes related to tumor-suppressor functions were more prevalent in $\mathrm{HPV}^{-} \mathrm{HNSCC}^{-}$ specimens. The observations may help to elucidate mechanisms involved in the molecular pathogenesis of two clinically diverse subclasses of HNSCC. Over-representation of SNPs in either $\mathrm{HPV}^{+}$or $\mathrm{HPV}^{-}$ HNSCC is another indicator of potentially actionable sequence variants for targeted therapy. (Am J Pathol 2014, 184: 1323-1330; http://dx.doi.org/10.1016/j.ajpath.2014.01.028)
\end{abstract}

Head and neck squamous cell carcinoma (HNSCC) is the sixth most common cancer worldwide. Despite recent advances in chemoradiation therapy, the 5-year survival rate for HNSCC is approximately 50\%. ${ }^{1}$ Based on epidemiology and disease outcomes, histologically diagnosed HNSCC has been stratified into two distinct prognostic entities directly linked to infection with high-risk human papilloma virus (HPV). Of the high-risk subtypes, HPV16 has been found in approximately $90 \%$ of infected HNSCC and HPV18 in approximately $5 \% .^{2-5}$ Other high-risk HPV types (eg, HPV31 and HPV33) have been detected in a few cases of HNSCC. ${ }^{2}$ The incidence of HPV-related oropharyngeal cancer continues to increase globally. ${ }^{6-9}$ Compared

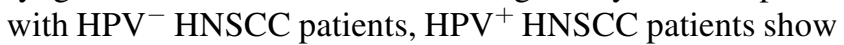
poor cell differentiation, better response to chemotherapy and radiotherapy, and better survival. ${ }^{2}$ The 3 -year survival of $\mathrm{HPV}^{+} \mathrm{HNSCC}$ patients is $83 \%$, as opposed to the $57 \%$ observed in $\mathrm{HPV}^{-}$HNSCC patients, and increased HPV viral copy number is associated with enhanced response to chemotherapy. ${ }^{10,11}$ In general, HPV infection and $\mathrm{p} 16^{\mathrm{INK} 4 \mathrm{a}}$ expression are recognized as predictive and prognostic biomarkers of HNSCC. ${ }^{12-15}$

Infection with HPV has divergent effects in different cancers; high-risk HPV16 and HPV18 subtypes account for $70 \%$ of cervical cancers with poor outcomes, whereas the virus has beneficial effects in patients with HNSCC. These differential

Current address, P.Z. and H.F., Department of Pathology and Laboratory Medicine, Weill Cornell Medical College, New York, NY

Disclosures: None declared. 
effects suggest that host elements that interact with the virus may play a role in determining the pathogenesis of the cancer and consequences of the infection. In the present study, we analyzed host factors that play a role in viral tumorigenesis, including the proinflammatory cytokine tumor necrosis factor alpha (TNF- $\alpha$ ) and the tumor suppressors $\mathrm{p} 16^{\mathrm{INK} 4 \mathrm{a}}$ (alias CDKN2A) and p53 (alias TP53). Others have shown that TP53 is the most commonly mutated gene in HNSCC and that this mutation is more prevalent in $\mathrm{HPV}^{-}$tumors. ${ }^{16}$ Genome-wide association studies using exome sequencing revealed that functionally disruptive mutations in genes such as $\mathrm{NOTCHI}$ are major drivers in the carcinogenesis of HNSCC. In a recent targeted next-generation sequencing (NGS) study, Lechner et $\mathrm{al}^{17}$ showed that genetic alterations in the PI3K pathway are more prevalent in $\mathrm{HPV}^{+}$HNSCC. The SNP profiles of HNSCC tumors differ between $\mathrm{HPV}^{+}$and $\mathrm{HPV}^{-}$subtypes. ${ }^{18}$

Using targeted NGS, we systematically analyzed somatic variants in HNSCC to further understand the differential genomic alterations in HPV-infected versus noninfected tumors. We compared the sequence variants of more than 2800 hotspots in 50 cancer-related genes present in $\mathrm{HPV}^{+}$and $\mathrm{HPV}^{-}$HNSCC patients in an effort to obtain fundamental insights into the pathogenesis of the disease that may identify potential diagnostic biomarkers and therapeutic targets.

\section{Materials and Methods}

\section{Patients}

Formalin-fixed, paraffin-embedded tissue blocks from 100 patients diagnosed with HNSCC between 2001 and 2013 were included in the study. Primary tumors from the tonsils, the base of the tongue, and the palate were selected for analysis. The sex and ethnicity distribution of the patients is summarized in Table 1. DNA from blood of eight healthy adults was included as an additional control in the sequencing analysis. The study was approved by the Institutional Review Board of Rutgers Biomedical and Health Sciences-New Jersey Medical School (protocol no. 0120100117).

\section{DNA Extraction}

The tumor-enriched area on H\&E-stained slides was demarcated by a pathologist (N.M.), and the corresponding area was identified on unstained tissue sections ( 4 to $5 \mu \mathrm{m}$ thick) taken from the same tissue block. The selected tissue was macrodissected by scraping it off the slides, and the tumor-enriched tissue was used for analysis. DNA from macrodissected tumor tissue $(n=100)$, matched nontumor tissue from HNSCC specimens $(n=8)$, and blood from healthy donors $(n=8)$ was extracted using a DNeasy blood and tissue kit (Qiagen, Valencia, CA) according to the manufacturer's recommendations. DNA was quantified using either a NanoDrop spectrophotometer (Thermo Fisher scientific, Waltham, MA) for PCR and for Sanger sequencing or a Qubit fluorometer (Life Technologies, Carlsbad, CA) for NGS.

\section{HPV Detection and Identification}

The presence of HPV16 and/or HPV18 was detected with a previously validated laboratory-developed test, using allelespecific quantitative real-time PCR on an ABI 7500 system (Life Technologies). ${ }^{19}$ Primers were synthesized at the Rutgers Molecular Resource Facility, and TaqMan probes were obtained from TIB MOLBIOL (Adelphia, NJ). Amplification of the $\beta$-globin gene as internal control was performed using a TaqMan Assay (Life Technologies). In brief, $2.5 \mu \mathrm{L}$ of extracted DNA, control DNA, or water (no-template control) was added to $22.5 \mu \mathrm{L}$ of master mix containing $1 \times$ TaqMan universal PCR master mix (Life Technologies), $2 \mathrm{mmol} / \mathrm{L} \mathrm{MgCl} 2,400 \mathrm{nmol} / \mathrm{L}$ of each primer, and $200 \mathrm{nmol} / \mathrm{L}$ TaqMan probe. Real-time amplification was performed using the following thermal profile: $50^{\circ} \mathrm{C}$ for 2 minutes and $95^{\circ} \mathrm{C}$ for 12 minutes, followed by 40 cycles of a three-step amplification at $95^{\circ} \mathrm{C}$ for 15 seconds, $55^{\circ} \mathrm{C}$ for 30 seconds, and $60^{\circ} \mathrm{C}$ for 60 seconds. Amplicons were sequenced to confirm the amplification of HPV16/18.

\section{Detection of p16 by Immunohistochemistry Staining}

Formalin-fixed, paraffin-embedded tissue sections were deparaffinized and subjected to antigen retrieval using $10 \mathrm{mmol} / \mathrm{L}$ citrate buffer $\left(92^{\circ} \mathrm{C}\right.$ for 30 minutes). Expression of p16 was evaluated using a mouse monoclonal antibody against $\mathrm{p} 16^{\mathrm{INK} 4 \mathrm{a}}$ (Cell Marque, Rocklin, CA) with 3,3'-diaminobenzidine as the chromogen. Specimens were scored as positive if strong and diffuse nuclear and cytoplasmic staining was present in $>70 \%$ of the tumor specimen (Supplemental Figure S1A).

\section{Detection of the -308 SNP in the TNFA Promoter}

DNA extracted from 53 HNSCC patient specimens (23 $\mathrm{HPV}^{+}$and $30 \mathrm{HPV}^{-}$) was analyzed for the $-308 \mathrm{TNFA}$ promoter single-nucleotide polymorphism (SNP) using Sanger sequencing (Life Technologies). Homozygous wildtype (GG), mutant (AA), and heterozygous (G/A) alleles were identified (Supplemental Figure S1B). Primers for sequencing were as described previously. ${ }^{20}$

\section{NGS}

A total of 69 specimens [53 HNSCC tumor samples (23 $\mathrm{HPV}^{+}$and $30 \mathrm{HPV}^{-}$), 8 matched nontumor specimens, and

Table 1 Sex and Ethnicity Distribution in 100 Patients

\begin{tabular}{lll}
\hline Demographics & Number of patients & Average age, years \\
\hline Sex & & \\
$\quad$ Female & 28 & 62.82 \\
$\quad$ Male & 72 & 58.39 \\
Ethnicity & & \\
$\quad$ Caucasian & 42 & 60.43 \\
Hispanic & 24 & 58.44 \\
African American & 20 & 56.8 \\
$\quad$ Other & 14 & 62.14 \\
Total & 100 & 59.07 \\
\hline
\end{tabular}


8 healthy donor samples] were subjected to NGS on an Ion Torrent PGM platform (Life Technologies). Libraries using $10 \mathrm{ng}$ of DNA were prepared with the Ion AmpliSeq $2.0 \mathrm{kit}$ (Life Technologies) according to the manufacturer's protocol. Genomic DNA was amplified in a multiplex PCR reaction using the Ion AmpliSeq Cancer Hotspot Panel v2, which targets 50 frequently mutated cancer genes and includes more than 2800 hotspots (ie, regions that are frequently mutated) (Supplemental Table S1). Details of the genomic positions for all hotspots are available from the manufacturer. Specimens were run in groups of four and were individually barcoded using an Ion Xpress barcode kit (Life Technologies).

The size distribution and quantity of DNA amplicons was evaluated using an Agilent 2100 bioanalyzer (Agilent Technologies, Santa Clara, CA). In brief, $1 \mu \mathrm{L}$ of prepared library was analyzed on an Agilent high-sensitivity dsDNA chip. A representative report generated by the Agilent bioanalyzer is shown in Supplemental Figure S1C. Peaks ranging from $100 \mathrm{bp}$ to $300 \mathrm{bp}$ were considered to be within the requisite amplicon size range. The concentration of amplicons in the library ranged from $1000 \mathrm{pmol} / \mathrm{L}$ to 5000 $\mathrm{pmol} / \mathrm{L}$. All libraries were then diluted appropriately to a final concentration of $20 \mathrm{pmol} / \mathrm{L}$. Equimolar amounts of four barcoded libraries that passed quality control were pooled, subjected to emulsion PCR using an Ion OneTouch 200 template kit version 2 DL (Life Technologies), and ligated to Ion Sphere beads. The quality of the amplicons and polyclonality was assessed using a Qubit fluorometer (Life Technologies). Amplicons were subsequently enriched to select viable nanospheres for sequencing on a 316 chip (Life Technologies). Enriched Ion Sphere particles were then loaded onto the 316 chip using an Ion PGM 200 sequencing kit according to the manufacturer's protocol (Life Technologies). Homogeneous loading of the 316 chip used for sequencing can be seen in the Supplemental Figure S1D.

Sequence reads generated were sorted into files and aligned to the human reference genome hg19 using Ion Torrent Variant Caller software version 3.4 (Life Technologies), and the Integrative Genomics Viewer (http:// www.broadinstitute.org/igv) was used to visualize variants. The visual picture of the sequence alignment with the reference as observed with the Integrative Genomics Viewer was used for elimination of false variants caused by possible sequencing errors or strand bias. Stringent criteria were applied for the determination of true variants. A sequencing run was considered acceptable if it had $\geq 150,000$ reads with a quality score of AQ20 (1 misaligned base per 100 bases). All gene variants reported in our study had to meet at least one of the following criteria: variant coverage $>1000 \times$ with $\geq 5 \%$ frequency, variant coverage $>500 \times$ and $\leq 1000 \times$ with $\geq 20 \%$ frequency, or variant coverage $>300 \times$ and $\leq 500 \times$ with $\geq 50 \%$ frequency. These conditions were set based on validation experiments performed in our laboratory.

\section{Statistical Analysis}

Fisher's exact test and $t$-test were used for statistical analysis.

\section{Results}

\section{HPV Infection in HNSCC and Correlation with p16}

Stratification of the 100 representative patients in the study (Table 1) showed that the number of men $(n=72)$ diagnosed histologically with HNSCC was roughly three times that of women $(n=28)$ in the study population. The average age was similar for both sexes. Analyzed by ethnicity, $42 \%$ of the patients were Caucasians, followed by Hispanics and African Americans. Overall, HPV was detected in 27/100 (27\%) tumor specimens analyzed (Table 1). Subtyping revealed that almost all of the 27 infected specimens had HPV16 (25/27), 1 had HPV18, and 1 was coinfected with both HPV16 and HPV18. Of the Caucasian patients, 16/42 (38\%) were $\mathrm{HPV}^{+}$, compared with 6/24 (25\%) for Hispanic patients and only $1 / 20(5 \%)$ for African American patients. The presence of p16 was examined by immunohistochemistry in all 100 patient specimens (Table 2), with a finding of $68 \%$ positive for p16 (CDKN2A). Representative images of stained slides are presented in Supplemental Figure S1A. There was a notable association between the presence of HPV and p16. Consistent with previous report, $23 \%$ of the specimens analyzed in our study were positive for both HPV and $\mathrm{p} 16 .^{12-15}$

\section{TNFA -308 Promoter Polymorphism and HNSCC}

We genotyped 53 patient specimens for the TNFA - 308 SNP by Sanger sequencing (Supplemental Figure S1B); 28.6\% of $\mathrm{HPV}^{+}$specimens were shown to have a proinflammatory $-308 \mathrm{~A}$ genotype, compared with $40 \%$ of $\mathrm{HPV}^{-}$specimens (Figure 1). We observed no significant association between the $-308 \mathrm{~A}$ allele and HPV infection in HNSCC $(P=0.34)$. Overall, $19(35.8 \%)$ of the 53 HNSCC patients genotyped had the -308 A allele, compared with $15.3 \%$ in the U.S. population (Centers for Disease Control and Prevention, http://www.cdc. gov/genomics/population/genvar/frequencies/TNF.htm, last accessed March 8, 2014).

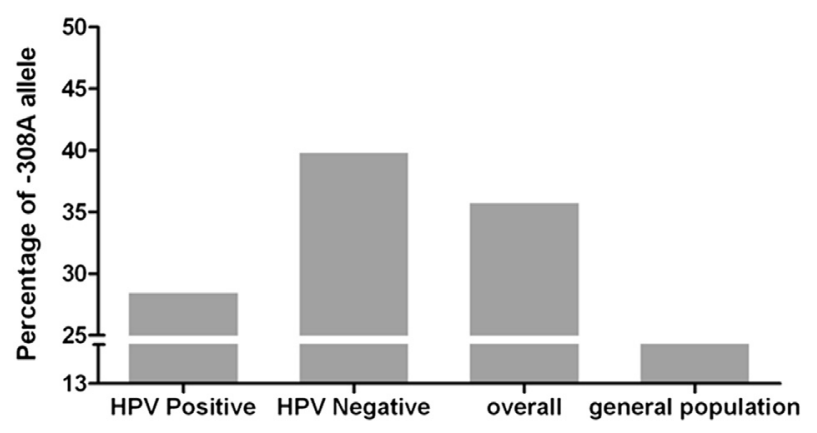

Figure 1 Percentage of TNFA -308 A allele in HNSCC patients. DNA extracted from HNSCC tissue specimens was subjected to Sanger sequencing using specific primers flanking the -308 SNP in the promoter region of the TNFA gene. The overall group represents combined $\mathrm{HPV}^{+}$and $\mathrm{HPV}^{-} \mathrm{HNSCC}$ specimens. 


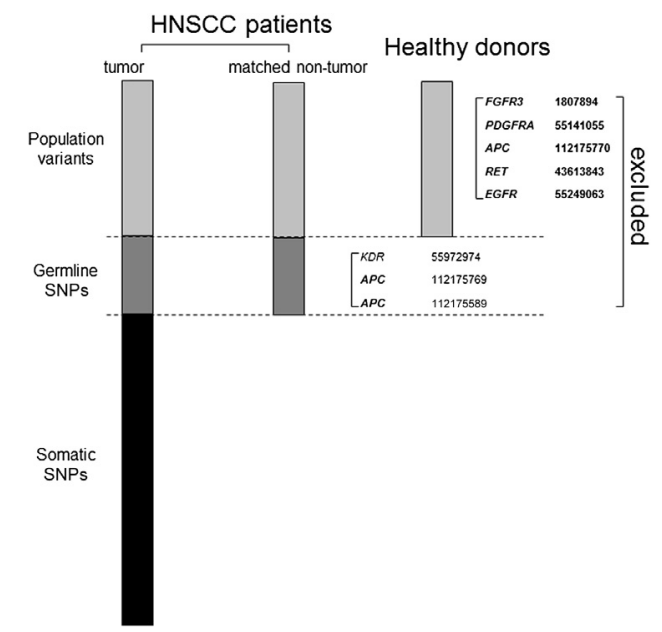

Figure 2 Types of variants detected in NGS analysis. Variants detected in NGS are classified as population variants, germline SNPs, and somatic SNPs based on the genetic alterations detected in healthy donors, patient-matched nontumor specimens, and HNSCC tumor specimens, respectively. Population variants and germline SNPs represent alterations that are not specific to HNSCC and therefore were excluded from further analysis.

\section{Genomic Variants in HNSCC Specimens Using Targeted NGS}

A total of 69 specimens [53 HNSCC specimens $\left(23 \mathrm{HPV}^{+}\right.$ and $\left.30 \mathrm{HPV}^{-}\right), 8$ matched nontumor specimens $\left(6 \mathrm{HPV}^{+}\right.$ and $2 \mathrm{HPV}^{-}$), and 8 healthy donor specimens] were subjected to targeted NGS to detect somatic variants in candidate cancer genes. Variants found in the specimens analyzed were categorized for clarification of overlapping patterns as population variants, germline SNPs, and somatic SNPs (Figure 2). Population variants were common to healthy donor samples and HNSCC patient samples, and these shared variants were excluded from further comparisons. Germline SNPs were variants detected in both tumor and nontumor components of HNSCC patients and therefore were not considered to be tumor-specific. Somatic variants, generally cancer related, were most often found in tumor tissue; these variants were the main focus of study.

\section{Genomic Variants in HNSCC Tumor, Matched Nontumor, and Healthy Donor Specimens}

SNPs in five different genes (FGFR3, chromosome position 1807894; PDGFRA, position 55141055; APC, position 112175770; RET, position 43613843; and EGFR, position $55249063)$ were present at high frequencies $(>90 \%)$ in all three specimen groups $(n=69)$ in the study (ie, 53 HNSCC specimens, 8 nontumor counterparts, and 8 healthy controls) (Figure 2). The presence of common or overlapping SNPs is suggestive of population variants, possibly unrelated to the disease or ethnicity, and therefore we excluded these five SNPs from further analysis.

\section{Germline SNPs in Tumor (HNSCC) versus Matched} Nontumor Specimens

Matched nontumor DNA from eight HNSCC specimens (six $\mathrm{HPV}^{+}$and two $\mathrm{HPV}^{-}$) was sequenced for identification of variants common to tumor and nontumor tissue. Data obtained from the Ion Torrent Variant Caller software was analyzed at the level of the gene and as individual sequence variants. Common SNPs in KDR (chromosome position 55972974) and $A P C$ (positions 112175769 and 112175589) were present in both tumor and nontumor DNA (Figure 2). Variants in other genes (eg, PIK3CA and MET) were also detected in both tumor and corresponding nontumor specimens. However, there was a notable difference in location of the individual variants within the PIK3CA gene. SNPs at four different hotspots of the PIK3CA gene present in tumor specimens were not detected in any of the matched nontumor HNSCC DNA samples. Likewise, SNPs at specific positions in the $M E T$ gene were seen only in the tumor DNA. One of the eight nontumor DNA specimens shared a SNP in the KIT gene with tumor DNA.

\section{Somatic SNPs in $\mathrm{HPV}^{+}$and $\mathrm{HPV}^{-}$HNSCC Tumor Specimens}

The average number of cancer-related genes that were mutated in $\mathrm{HPV}^{-}$specimens was 3.23, compared with 2.16 in $\mathrm{HPV}^{+}$specimens (Figure 3). The difference was not significant $(P=0.069)$.

Somatic variants in five candidate genes were found in both $\mathrm{HPV}^{+}$and $\mathrm{HPV}^{-}$HNSCC specimens: PDGFRA, PIK3CA, KIT, MET, and APC (Figure 4). Although variants from four of the five genes appeared to be more frequent in $\mathrm{HPV}^{+}$than in $\mathrm{HPV}^{-}$specimens, the difference was not statistically significant. Variants in PDGFRA were present in approximately $30.43 \%$ of $\mathrm{HPV}^{+}$HNSCC specimens, compared with $16.67 \%$ in $\mathrm{HPV}^{-}$HNSCC specimens, indicating a possible association of this gene with HPV in HNSCC $(P=0.52)$. Individual SNPs in the KIT gene of $\mathrm{HPV}^{-}$specimens were restricted to a single chromosome position (55593464), compared with four different positions detected in $\mathrm{HPV}^{+}$specimens. Likewise, $75 \%$ of the SNPs in the $M E T$ gene of $\mathrm{HPV}^{-}$specimens were found at a single

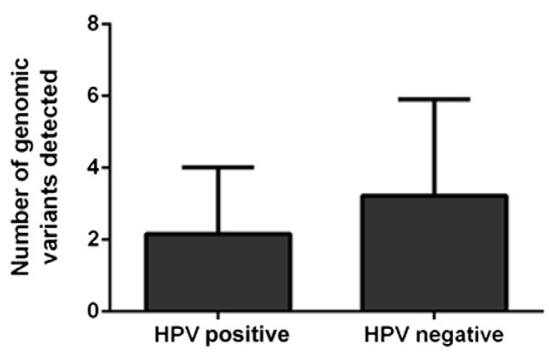

Figure 3 Number of variants found in HNSCC patients. HNSCC specimens $(n=53)$ determined to be $\mathrm{HPV}^{+}(n=23)$ or $\mathrm{HPV}^{-}(n=30)$ were examined for SNPs in 50 cancer-related genes using NGS. Data are expressed as means \pm SEM. 


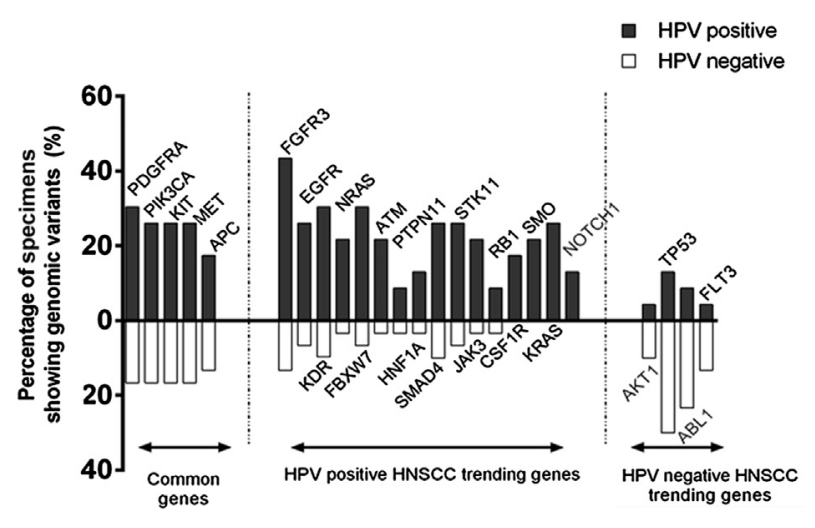

Figure 4 Summary of genomic variants in HNSCC patients. Genes were categorized as common gene variants in $\mathrm{HPV}^{+}$and $\mathrm{HPV}^{-} \mathrm{HNSCC}_{1} \mathrm{HPV}^{+}$ trending genes, and $\mathrm{HPV}^{-}$trending genes.

position (116339672), whereas the SNPs in $\mathrm{HPV}^{+}$specimens were detected at three positions (116339673, 116411973, and 116411978).

Sequence variants in 16 genes were present at higher frequency in $\mathrm{HPV}^{+} \mathrm{HNSCC}$ patients than in $\mathrm{HPV}^{-}$patients: FGFR3, EGFR, KDR, NRAS, FBXW7, CSF1R, SMO, ATM, PTPN11, HNF1A, SMAD4, STK11, JAK3, KRAS, NOTCH1, and $R B 1$. The position of variants in these genes differed between $\mathrm{HPV}^{+}$and $\mathrm{HPV}^{-}$specimens. FGFR3 $(P=0.02)$, NRAS $(P=0.03)$, and $F B X W 7(P=0.03)$ variants were detected in $43.5 \%, 21.74 \%$, and $30.43 \%$ of $\mathrm{HPV}^{+}$specimens, respectively, which is significantly greater than the corresponding $13.3 \%, 3.3 \%$, and $6.7 \%$ in $\mathrm{HPV}^{-}$specimens. Furthermore, variants in CSFIR $(P=0.03)$, SMO $(P=0.01)$, and $\operatorname{KRAS}(P=0.004)$ were found exclusively in $\mathrm{HPV}^{+}$specimens, suggesting that the SNPs in these genes may be substantially associated with HPV infection in HNSCC. NOTCH1 variants were also present exclusively in $\mathrm{HPV}^{+}$specimens. The ATM variants that were present did not show any statistical difference between the two groups
$(P=0.074)$. For $H R A S$, only one variant was detected, in an $\mathrm{HPV}^{+}$specimen. Differences in frequency between $\mathrm{HPV}^{+}$ and $\mathrm{HPV}^{-}$HNSCC for SNPs in EGFR, KDR, and SMAD4 $(P=0.07, P=0.08$, and $P=0.15)$ were not significant.

Four genes were altered primarily in $\mathrm{HPV}^{-} \mathrm{HNSCC}$ specimens: AKT1, TP53, ABL1, and FLT3 (Figure 4). There was no statistical difference in these genes in $\mathrm{HPV}^{+}$and $\mathrm{HPV}^{-}$specimens.

A summary of the genomic variants is presented in Figure 4. The common genes represent population variants distributed similarly in both $\mathrm{HPV}^{+}$and $\mathrm{HPV}^{-}$HNSCC. By contrast, genomic variants occur preferentially in $\mathrm{HPV}^{+}$ versus $\mathrm{HPV}^{-}$HNSCC.

\section{Discussion}

In contrast to HPV infections of the genital tract, disease progression of HNSCC has a more favorable outcome if the tumor is infected with the high-risk HPV16 and/or HPV18 subtype. The differential effects of the virus in different cancers implies that a combination of viral and host risk factors play an important role in the oncogenesis of these cancers. In the present study, we identified HPV16 in $92.6 \%$ of $\mathrm{HPV}^{+}$ tumors, compared with $68 \%$ to $87 \%$ in the literature. ${ }^{1,5}$ The difference may be attributed to the fact that we studied only the HPV16 and HPV18 high-risk subtypes. The higher incidence of HPV infection in men than in women in our study population is in keeping with demographic data for HNSCC. ${ }^{18}$ This apparent difference is explained, at least in part, by associated risk factors such as tobacco and alcohol usage and sexual practices. ${ }^{2,19}$ In agreement with previous report, ${ }^{19}$ in the present study $68 \%$ of the HNSCC specimens were positive for p16 and 33.8\% (23/68) were also positive for HPV.

Overall, the sequencing data showed key differences in the genetic alterations identified in the cancer-related genes

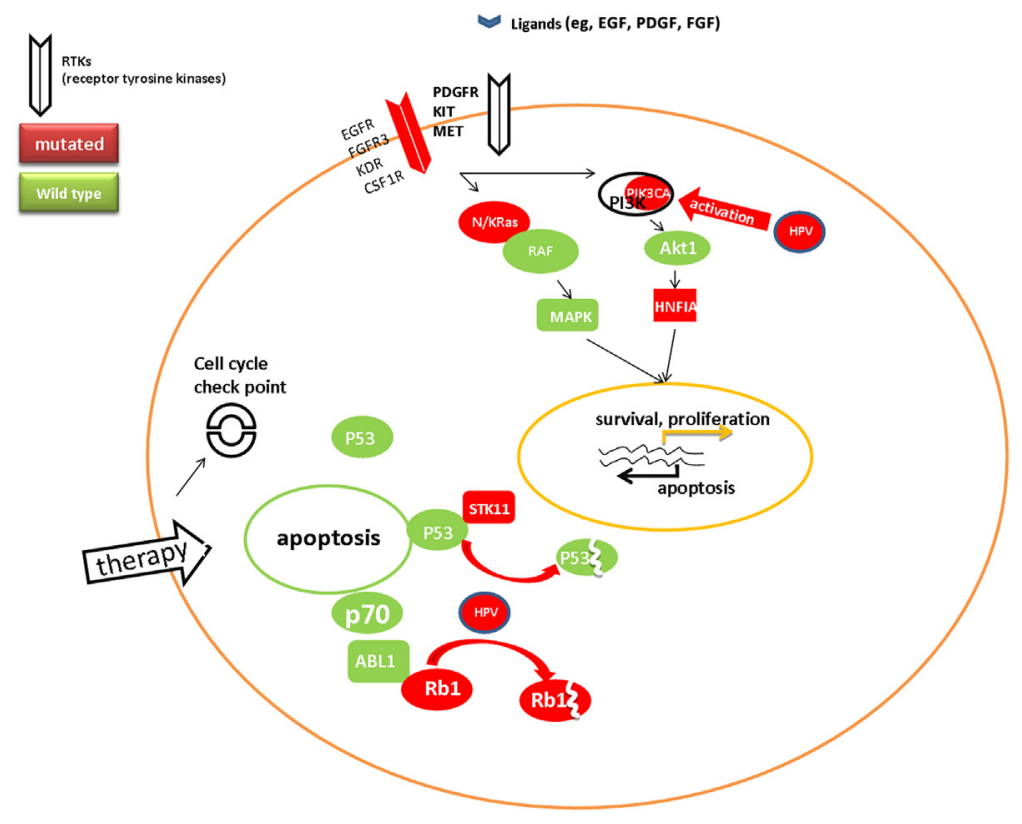

Figure 5 Proposed pathway involved in pathogenesis of $\mathrm{HPV}^{+}$HNSCC. In $\mathrm{HPV}^{+} \mathrm{HNSCC}$, several genes for RTK receptors and their downstream pathways are found to be altered, leading to increased cell proliferation and eventually resulting in development of cancer. The majority of $\mathrm{HPV}^{+} \mathrm{HNSCC}$ patients have wild-type $\mathrm{p53}$, which can induce cell apoptosis in response to chemotherapy. Symbols for molecules do not scale to molecular properties. 


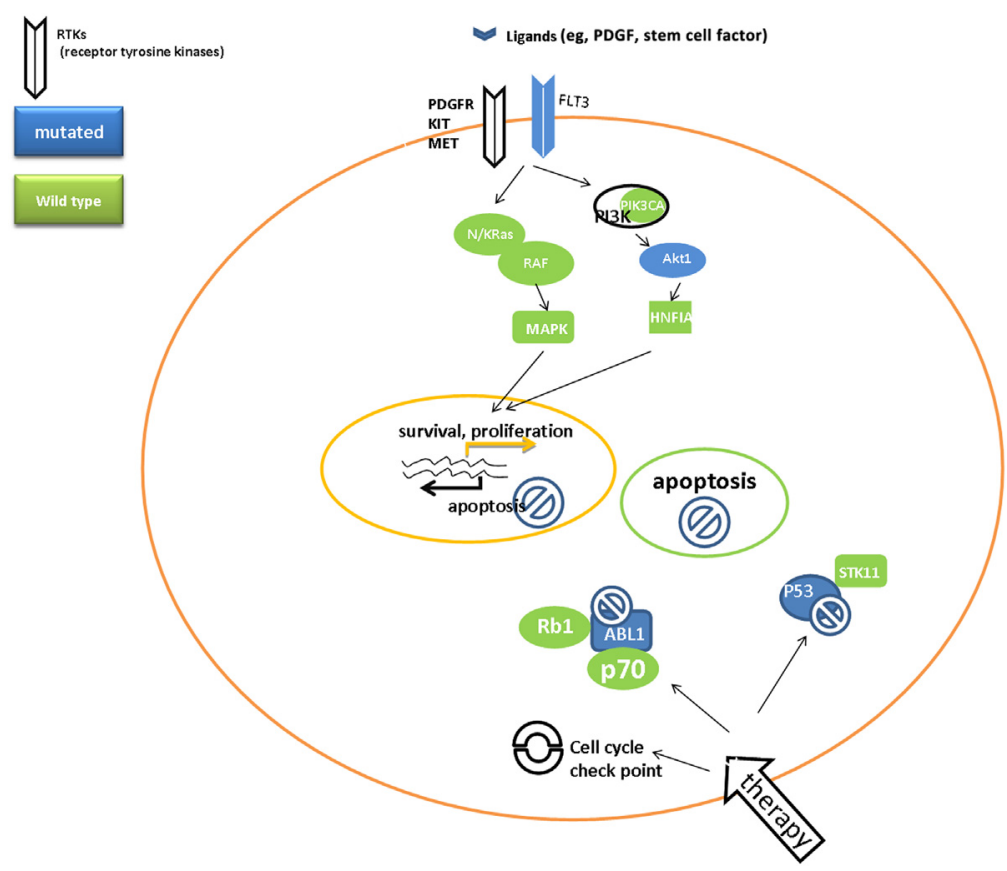

Figure 6 Proposed pathway involved in pathogenesis of $\mathrm{HPV}^{-}$HNSCC. In HPV ${ }^{-}$HNSCC, several RTK receptors are altered, but with fewer downstream variants than for $\mathrm{HPV}^{+}$ HNSCC. More importantly, a much higher percentage of TP53 and $A B L 1$ variants are associated with $\mathrm{HPV}^{-}$HNSCC, possibly leading to cancer development and poor response to therapy. Symbols for molecules do not scale to molecular properties.

and signaling pathways of $\mathrm{HPV}^{+}$and $\mathrm{HPV}^{-} \mathrm{HNSCC}^{- \text {Our }}$ analysis showed a higher incidence of the TNFA proinflammatory -308A allele $(37.2 \%)$ in HNSCC patients, irrespective of HPV infection, compared with the documented allele frequency in different ethnic groups. ${ }^{21-24}$ This observation corroborates earlier report of an association between the -308 TNFA SNP and HNSCC in a European cohort $^{25}$ and points toward a potential role for TNF- $\alpha$ as a risk factor in the pathogenesis of HNSCC.

Targeted NGS analysis of the genes involved in growthfactor signaling showed that a number of genes from the receptor tyrosine kinase (RTK) family and their associated pathways are altered in HNSCC. Sequence variants in genes encoding RTK receptors (eg, EGFR, FGFR3, PDGFR, $M E T, K D R, C S F 1 R$, and $K I T$ ) were preferentially associated with $\mathrm{HPV}^{+} \mathrm{HNSCC}$ specimens (Figure 5). In particular, $\mathrm{HPV}^{+}$tumors exhibited a significantly higher frequency of FGFR3 variants $(P=0.02)$ and an exclusive presence of $C S F 1 R$ variants $(P=0.03)$. The importance of RTK pathway variants in oncology and their relevance to responsiveness with targeted therapy has been highlighted in several cancers, including HNSCC. However, monoclonal antibodies directed toward the EGFR receptor, smallmolecule tyrosine kinase inhibitors (TKIs) that bind the catalytic kinase domain of the receptor, and TKIs that target multiple tyrosine kinase receptors have met with limited success in HNSCC. ${ }^{26}$ Recent in vitro data from Singleton et $\mathrm{al}^{27}$ support combinations of TKIs targeting FGFR, ERBB family members, and $M E T$ for more effective therapeutic management of HNSCC. Our findings show that variants in these genes are more frequent in the clinically favorable $\mathrm{HPV}^{+} \mathrm{HNSCC}$. It is possible that variants in the RTK receptors seen in $\mathrm{HPV}^{+} \mathrm{HNSCC}$ could potentially interfere with downstream signaling to control proliferation.

Two classic pathways downstream of RTKs that are activated on receptor dimerization are the RAS-MAPK and

Table 2 HPV Infection and Presence of p16 in 100 HNSCC Patients

\begin{tabular}{|c|c|c|c|c|c|c|}
\hline \multirow[b]{2}{*}{ Demographics } & \multirow[b]{2}{*}{ Sample size } & \multicolumn{5}{|c|}{ Patients [no. (\%)] } \\
\hline & & HPV16 & HPV18 & $\mathrm{HPV}^{-}$ & $\mathrm{p} 16^{+}$ & $\mathrm{p} 16^{+} \mathrm{HPV}^{+}$ \\
\hline \multicolumn{7}{|l|}{ Sex } \\
\hline Female & 28 & $8(28.6)$ & $0(0)$ & $20(71.4)$ & $20(71.4)$ & $8(28.6)$ \\
\hline Male & 72 & $18(25)$ & $2^{*}(2.8)$ & $53(72.6)$ & $48(68.1)$ & $15(19.4)$ \\
\hline \multicolumn{7}{|l|}{ Ethnicity } \\
\hline Caucasian & 42 & $15(35.7)$ & $1(2.4)$ & $26(61.9)$ & $30(71.4)$ & $13(33.3)$ \\
\hline Hispanic & 24 & $6(25)$ & $1^{*}(4.2)$ & $18(75)$ & $17(70.8)$ & $6(25)$ \\
\hline African American & 20 & $1(5)$ & $0(0)$ & $19(95)$ & $11(55)$ & $1(5)$ \\
\hline Other & 14 & $3(21.4)$ & 0 & $11(78.6)$ & $10(71.4)$ & $3(21.4)$ \\
\hline Total & 100 & $26(26)$ & $2^{*}(2)$ & $73(73)$ & $68(68)$ & $23(23)$ \\
\hline
\end{tabular}

${ }^{*}$ One patient was double positive for both HPV16 and HPV18. 
PI3K-AKT pathways. The RAS isoforms collectively are among the most mutated genes in HNSCC patients, and there is a strong association between the presence of KRAS variants and tobacco-related cancers, including HNSCC. ${ }^{28}$ Our results show an increased number of SNPs in NRAS and the presence of KRAS sequence variants (including the well-characterized activating mutation $\mathrm{G} 12 \mathrm{~V}$ ) primarily in $\mathrm{HPV}^{+}$specimens. Interestingly, mutations at codon 12 of the KRAS genes that lead to constitutional activation of $E G F R$ have been detected previously in HNSCC, but were not associated with metastatic potential. ${ }^{29,30}$

Missense mutations and copy number alterations in the PIK3CA gene have been previously implicated in the carcinogenesis of HNSCC. ${ }^{30,31}$ Lechner et $\mathrm{al}^{17}$ reported a high frequency of $\mathrm{PI} 3 \mathrm{~K}$ pathway activation in $\mathrm{HPV}^{+}$ HNSCC, and data from the Cancer Genome Atlas research network suggest that the high mutation rate of PIK3CA in HPV-infected cells makes it a more appropriate target than $E G F R$ for the treatment of patients with HNSCC. ${ }^{32}$ Our studies have documented the presence of PIK3CA variants in both $\mathrm{HPV}^{+}$and $\mathrm{HPV}^{-}$patients, supporting the hypothesis that PIK3CA may be a viable target for HNSCC. Additionally, the presence of $A K T 1$ variants in our patient cohort corroborates the involvement of the PI3K-AKT pathway in the pathogenesis and progression of HNSCC. As observed previously, we also demonstrated specific sequence variations in STK11 in $\mathrm{HPV}^{+} \mathrm{HNSCC}^{17,33}$ Lossof-function variants in the tumor suppressor STK11 gene are associated with tumorigenesis of sporadic and metastatic HNSCC. $^{33}$ Because PIK3CA and STK11 regulate cell growth by modulating the mammalian target of rapamycin complex 1 (mTORC1) pathway, the PI3K-AKT pathway appears to be a target of interest in oropharyngeal cancer.

HNSCC tumor cells responsive to chemotherapeutic agents such as cisplatin undergo apoptosis mediated by $\mathrm{p} 53$, RB1, and other facilitator molecules (eg, ABL1). Variants in genes (TP53 and ABL1) encoding these proteins are overrepresented in the $\mathrm{HPV}^{-} \mathrm{HNSCC}$ specimens analyzed, suggesting that increased levels of the altered protein may result in the lack of apoptosis in the tumor (Figure 6). These findings are consistent with previous observations that wildtype genes encoding p53 and RB1 predominantly present in $\mathrm{HPV}^{+} \mathrm{HNSCC}$ are able to produce functional proteins, thus leading to a better prognosis. ${ }^{34}$ In this regard, the allelic substitution at codon 72 of the TP53 gene (CGC-arginine or CCC-proline) that plays a major role in inducing apoptosis in TP53 mutant cells ${ }^{35,36}$ merits further investigation as a target for therapy (Figure 6).

In addition to the genes involved in signaling for apoptosis and survival, variants observed in the $F B X W 7$ and $S M O$ genes of the hedgehog pathway may be also involved in development of HNSCC with HPV infection. A recent report highlights differences in the variant landscape of HNSCC, particularly TP53, FBXW7, and NOTCH1, related to the tumor site and the patient's ethnicity. ${ }^{37} \mathrm{We}$, however, did not observe any significant variants in the NOTCHI or
$C D K N 2 A$ genes. A limitation of the present study is the use of targeted analysis, which provides information limited to the interrogated area of the gene (in contrast to exome sequencing, which can provide additional data related to the entire genome).

In summary, we have identified specific genetic variants that provide potential insight into the differential pathogenesis of $\mathrm{HPV}^{+}$and $\mathrm{HPV}^{-}$HNSCC. Previous studies to differentiate $\mathrm{HPV}^{+}$from $\mathrm{HPV}^{-}$HNSCC addressed primarily $T P 53$ and $E G F R$ gene variants. Our present findings confirm the importance of other genes (eg, PIK3CA), add to knowledge of SNPs in STK11 and FBXW7, and point out differences in SNP profiles of RTK pathway genes (eg, $F G F R 3, K R A S$, and $C S F 1 R$ ) in $\mathrm{HPV}^{+}$and $\mathrm{HPV}^{-} \mathrm{HNSCC}$. Several of the genes identified here have been speculated to be either driver or candidate targets for therapy in HNSCC by the Cancer Genome Atlas project. ${ }^{32}$ The diversity of SNP profiles seen in HNSCC patients highlights the complex nature of the disease and emphasizes the importance of improved patient selection using comprehensive variant analyses for the most effective targeted therapies.

\section{Acknowledgments}

We thank Anjali Seth and Madhuri Ramanathan for their expertise in validating the assays for the identification of HPV16 and HPV18 and thank Dr. Robert Donnelly for his valuable suggestions.

\section{Supplemental Data}

Supplemental material for this article can be found at http://dx.doi.org/10.1016/j.ajpath.2014.01.028.

\section{References}

1. Leemans CR, Braakhuis BJ, Brakenhoff RH: The molecular biology of head and neck cancer. Nat Rev Cancer 2011, 11:9-22

2. Zandberg DP, Bhargava R, Badin S, Cullen KJ: The role of human papillomavirus in nongenital cancers. CA Cancer J Clin 2013, 63: $57-81$

3. Morbini P, Dal Bello B, Alberizzi P, Mannarini L, Mevio N, Bertino G, Benazzo M: Exfoliated cells of the oral mucosa for HPV typing by SPF10 in head and neck cancer. J Virol Methods 2012, 186:99-103

4. de Jong MC, Pramana J, Knegjens JL, Balm AJ, van den Brekel MW, Hauptmann M, Begg AC, Rasch CR: HPV and high-risk gene expression profiles predict response to chemoradiotherapy in head and neck cancer, independent of clinical factors. Radiother Oncol 2010, 95: $365-370$

5. D'Souza G, Dempsey A: The role of HPV in head and neck cancer and review of the HPV vaccine. Prev Med 2011, 53(Suppl 1):S5-S11

6. Kreimer AR, Clifford GM, Boyle P, Franceschi S: Human papillomavirus types in head and neck squamous cell carcinomas worldwide: a systematic review. Cancer Epidemiol Biomarkers Prev 2005, 14: 467-475

7. Kreimer AR, Clifford GM, Snijders PJ, Castellsague X, Meijer CJ, Pawlita M, Viscidi R, Herrero R, Franceschi S; International Agency for Research on Cancer (IARC) Multicenter Oral Cancer Study Group: HPV16 semiquantitative viral load and serologic biomarkers in oral 
and oropharyngeal squamous cell carcinomas. Int J Cancer 2005, 115 329-332

8. D'Souza G, Kreimer AR, Viscidi R, Pawlita M, Fakhry C, Koch WM, Westra WH, Gillison ML: Case-control study of human papillomavirus and oropharyngeal cancer. N Engl J Med 2007, 356:1944-1956

9. Nasman A, Attner P, Hammarstedt L, Du J, Eriksson M, Giraud G, Ahrlund-Richter S, Marklund L, Romanitan M, Lindquist D, Ramqvist T, Lindholm J, Sparen P, Ye W, Dahlstrand H, MunckWikland E, Dalianis T: Incidence of human papillomavirus (HPV) positive tonsillar carcinoma in Stockholm, Sweden: an epidemic of viral-induced carcinoma? Int J Cancer 2009, 125:362-366

10. Ang KK, Harris J, Wheeler R, Weber R, Rosenthal DI, NguyenTan PF, Westra WH, Chung CH, Jordan RC, Lu C, Kim H, Axelrod R, Silverman CC, Redmond KP, Gillison ML: Human papillomavirus and survival of patients with oropharyngeal cancer. New Engl J Med 2010, 363:24-35

11. Worden FP, Kumar B, Lee JS, Wolf GT, Cordell KG, Taylor JM, Urba SG, Eisbruch A, Teknos TN, Chepeha DB, Prince ME, Tsien CI, D'Silva NJ, Yang K, Kurnit DM, Mason HL, Miller TH, Wallace NE, Bradford CR, Carey TE: Chemoselection as a strategy for organ preservation in advanced oropharynx cancer: response and survival positively associated with HPV16 copy number. J Clin Oncol 2008, 26:3138-3146

12. Lassen P, Eriksen JG, Hamilton-Dutoit S, Tramm T, Alsner J, Overgaard J: Effect of HPV-associated p16INK4A expression on response to radiotherapy and survival in squamous cell carcinoma of the head and neck. J Clin Oncol 2009, 27:1992-1998

13. van den Broek GB, Wildeman $\mathrm{M}$, Rasch $\mathrm{CR}$, Armstrong $\mathrm{N}$, Schuuring E, Begg AC, Looijenga LH, Scheper R, van der Wal JE, Menkema L, van Diest PJ, Balm AJ, van Velthuysen ML, van den Brekel MW: Molecular markers predict outcome in squamous cell carcinoma of the head and neck after concomitant cisplatin-based chemoradiation. Int J Cancer 2009, 124:2643-2650

14. Rainsbury JW, Ahmed W, Williams HK, Roberts S, Paleri V, Mehanna H: Prognostic biomarkers of survival in oropharyngeal squamous cell carcinoma: systematic review and meta-analysis. Head Neck 2013, 35:1048-1055

15. Lassen P, Eriksen JG, Hamilton-Dutoit S, Tramm T, Alsner J, Overgaard J; Danish Head and Neck Cancer Group (DAHANCA): HPV-associated p16-expression and response to hypoxic modification of radiotherapy in head and neck cancer. Radiother Oncol 2010, 94: $30-35$

16. Braakhuis B, Rietbergen M, Buijze M, Snijders P, Bloemena E, Brakenhoff R, Leemans C: TP53 mutation and human papilloma virus status of oral squamous cell carcinomas in young adult patients. Oral Dis 2013, [Epub ahead of print] http://dx.doi.org/10.1111/odi.12178

17. Lechner M, Frampton GM, Fenton T, Feber A, Palmer G, Jay A, Pillay N, Forster M, Cronin MT, Lipson D, Miller VA, Brennan TA, Henderson S, Vaz F, O’Flynn P, Kalavrezos N, Yelensky R, Beck S, Stephens PJ, Boshoff C: Targeted next-generation sequencing of head and neck squamous cell carcinoma identifies novel genetic alterations in HPV + and HPV- tumors. Genome Med 2013, 5:49

18. Stransky N, Egloff AM, Tward AD, Kostic AD, Cibulskis K, Sivachenko A, et al: The mutational landscape of head and neck squamous cell carcinoma. Science 2011, 333:1157-1160

19. Gravitt PE, Peyton C, Wheeler C, Apple R, Higuchi R, Shah KV: Reproducibility of HPV 16 and HPV 18 viral load quantitation using TaqMan real-time PCR assays. J Virol Methods 2003, 112:23-33

20. Fernandes H, Koneru B, Fernandes N, Hameed M, Cohen MC, Raveche E, Cohen S: Investigation of promoter polymorphisms in the tumor necrosis factor-alpha and interleukin-10 genes in liver transplant patients. Transplantation 2002, 73:1886-1891

21. Conway DJ, Holland MJ, Bailey RL, Campbell AE, Mahdi OS, Jennings R, Mbena E, Mabey DC: Scarring trachoma is associated with polymorphism in the tumor necrosis factor alpha (TNF-alpha) gene promoter and with elevated TNF-alpha levels in tear fluid. Infect Immun 1997, 65:1003-1006
22. Mozzato-Chamay N, Mahdi OS, Jallow O, Mabey DC, Bailey RL, Conway DJ: Polymorphisms in candidate genes and risk of scarring trachoma in a Chlamydia trachomatis-endemic population. J Infect Dis 2000, 182:1545-1548

23. Keatings VM, Cave SJ, Henry MJ, Morgan K, O’Connor CM, FitzGerald MX, Kalsheker N: A polymorphism in the tumor necrosis factor-alpha gene promoter region may predispose to a poor prognosis in COPD. Chest 2000, 118:971-975

24. Cuenca J, Cuchacovich M, Pérez C, Ferreira L, Aguirre A, Schiattino I, Soto L, Cruzat A, Salazar-Onfray F, Aguillon JC: The -308 polymorphism in the tumour necrosis factor (TNF) gene promoter region and ex vivo lipopolysaccharide-induced TNF expression and cytotoxic activity in Chilean patients with rheumatoid arthritis. Rheumatology 2003, 42:308-313

25. Yapijakis C, Serefoglou Z, Vylliotis A, Nkenke E, Derka S, Vassiliou S, Avgoustidis D, Neukam FW, Patsouris E, Vairaktaris E: Association of polymorphisms in tumor necrosis factor alpha and beta genes with increased risk for oral cancer. Anticancer Res 2009, 29:2379-2386

26. Rikiishi $\mathrm{H}$ : Autophagic action of new targeting agents in head and neck oncology. Cancer Biol Ther 2012, 13:978-991

27. Singleton KR, Kim J, Hinz TK, Marek LA, Casas-Selves M, Hatheway C, Tan AC, DeGregori J, Heasley LE: A receptor tyrosine kinase network composed of fibroblast growth factor receptors, epidermal growth factor receptor, v-erb-b2 erythroblastic leukemia viral oncogene homolog 2 , and hepatocyte growth factor receptor drives growth and survival of head and neck squamous carcinoma cell lines. Mol Pharmacol 2013, 83:882-893

28. Murugan AK, Munirajan AK, Tsuchida N: Ras oncogenes in oral cancer: the past 20 years. Oral Oncol 2012, 48:383-392

29. Bissada E, Abboud O, Abou Chacra Z, Guertin L, Weng X, NguyenTan PF, Tabet JC, Thibaudeau E, Lambert L, Audet ML, Fortin B, Soulieres D: Prevalence of K-RAS codons 12 and 13 mutations in locally advanced head and neck squamous cell carcinoma and impact on clinical outcomes. Int J Otolaryngol 2013, 2013:848021

30. Suda T, Hama T, Kondo S, Yuza Y, Yoshikawa M, Urashima M, Kato T, Moriyama H: Copy number amplification of the PIK3CA gene is associated with poor prognosis in non-lymph node metastatic head and neck squamous cell carcinoma. BMC Cancer 2012, 12:416

31. Du L, Shen J, Weems A, Lu SL: Role of phosphatidylinositol-3-kinase pathway in head and neck squamous cell carcinoma. J Oncol 2012, 2012:450179

32. Nelson NJ: Genetic events in head and neck squamous cell carcinoma revealed. J Natl Cancer Inst 2013, 105:1766-1768

33. Qiu W, Schönleben F, Thaker HM, Goggins M, Su GH: A novel mutation of STK11/LKB1 gene leads to the loss of cell growth inhibition in head and neck squamous cell carcinoma. Oncogene 2006, 25: $2937-2942$

34. Peltonen JK, Vähäkangas KH, Helppi HM, Bloigu R, Pääkko P, Turpeenniemi-Hujanen T: Specific TP53 mutations predict aggressive phenotype in head and neck squamous cell carcinoma: a retrospective archival study. Head Neck Oncol 2011, 3:20

35. Schneider-Stock R, Boltze C, Peters B, Szibor R, Landt O, Meyer F, Roessner A: Selective loss of codon 72 proline p53 and frequent mutational inactivation of the retained arginine allele in colorectal cancer. Neoplasia 2004, 6:529-535

36. Mozet C, Stoehr M, Dimitrova K, Dietz A, Wichmann G: Hedgehog targeting by cyclopamine suppresses head and neck squamous cell carcinoma and enhances chemotherapeutic effects. Anticancer Res 2013, 33:2415-2424

37. Guerrero-Preston R, Hadar T, Michailidi C, Marchionni L, Koch W, Sidransky D: Integrated genomic and epigenomic deep sequencing analyses reveal head and neck cancer survival disparities associated to alterations in the PAX, NOTCH1 and TP53 pathways. 6th AACR Conference on the Science of Cancer Health Disparities in Racial/ Ethnic Minorities and the Medically Underserved, Dec. 6-9, 2013, Atlanta GA. Philadelphia, American Association for Cancer Research, 2013, Abstract A87 